\title{
A NOTE ON A CHARACTERIZATION OF METRICS GENERATED BY NORMS
}

\author{
JACEK CHMIELIŃSKI
}

\begin{abstract}
With reference to papers of Oikhberg and Rosenthal [3] and Šemrl [4, 5], we make a contribution to the problem of characterization of metrics generated by norms.
\end{abstract}

1. Introduction. Let $X$ be a real linear space with a metric $d$ on it. Šemrl [5] (motivated by earlier papers $[\mathbf{3}, \mathbf{4}]$ ) showed that $d$ is generated by a norm if and only if: it is translation invariant

$$
d(x+z, y+z)=d(x, y), \quad x, y, z \in X,
$$

algebraic midpoints are metric ones

$$
d\left(\frac{x+y}{2}, x\right)=d\left(\frac{x+y}{2}, y\right)=\frac{1}{2} d(x, y), \quad x, y \in X
$$

and

$$
\text { for each } x \in X \text { the set }\{t x: t \in[0,1]\} \text { is bounded. }
$$

Conditions (1.1) and (1.2) do not suffice, for consider the metric $d_{f}$ in $\mathbb{R}$ where $d_{f}(x, y)=|f(x)-f(y)|$ and $f: \mathbb{R} \rightarrow \mathbb{R}$ is an injective and discontinuous additive mapping.

Analyzing the proof of Šemrl's theorem one can notice that, in fact, condition (1.2) can be relaxed to

$$
d(2 x, 0)=2 d(x, 0), \quad x \in X .
$$

2010 AMS Mathematics subject classification. Primary 39B52, 39B62, 46B20, $54 \mathrm{E} 35$.

Keywords and phrases. Metrics generated by norms, additive mappings, convex mappings.

Received by the editors on January 26, 2014, and in revised form on March 7, 2014. 
The conjunction of (1.1) and (1.2) is equivalent to that of (1.1) and $\left(1.2^{\prime}\right)$. Condition $\left(1.2^{\prime}\right)$ is essentially weaker than (1.2), and it is easy to observe that neither $\left(1.2^{\prime}\right)$ implies (1.1), nor (1.1) implies $\left(1.2^{\prime}\right)$.

The aim of this note is to consider another geometrical property of a metric, which together with some regularity condition characterizes normed spaces among metric ones.

\section{Mid-segment property and a characterization of normed} spaces. Let us consider a condition which relates to the elementary mid-segment property: if $x, y$ and $z$ are vertices of a triangle, then the segment joining the midpoints $(x+z) / 2$ and $(y+z) / 2$ is half as long as the one joining $x$ and $y$. Thus, let

$$
d\left(\frac{x+z}{2}, \frac{y+z}{2}\right)=\frac{1}{2} d(x, y), \quad x, y, z \in X,
$$

which equivalently could be written in a simpler form:

$$
d\left(\frac{x-y}{2}, 0\right)=\frac{1}{2} d(x, y), \quad x, y \in X .
$$

Condition $(*)$ is equivalent to the conjunction of conditions (1.1) and (1.2). Indeed, it follows from $(*)$ that $d(x / 2,0)=d(x, 0) / 2$, whence $d(x, y)=d(x-y, 0)$. Inserting $x+z$ and $y+z$ in place of $x$ and $y$ one gets (1.1). Next, using (1.1), we have

$$
d\left(\frac{x+y}{2}, y\right)=d\left(\frac{x-y}{2}+y, y\right)=d\left(\frac{x-y}{2}, 0\right)=\frac{1}{2} d(x, y),
$$

whence (1.2) holds true. Conversely, assuming (1.1) and (1.2), one gets $(*)$ :

$$
d\left(\frac{x-y}{2}, 0\right)=d\left(\frac{x-y}{2}+y, y\right)=d\left(\frac{x+y}{2}, y\right)=\frac{1}{2} d(x, y) .
$$

It follows thus from the theorem of Šemrl that any metric $d$ satisfying $(*)$ and (1.3) comes from a norm and conversely. Assuming merely $(*)$, we obtain a somewhat weaker result.

Proposition 2.1. If a metric $d$ on a real vector space $X$ satisfies $(*)$, then there exists a mapping $\varphi: X \rightarrow[0, \infty)$ satisfying conditions: 
(i) $\varphi(x)=0 \Leftrightarrow x=0$;

(ii) $\varphi(-x)=\varphi(x), x \in X$;

(iii) $\varphi(x+y) \leq \varphi(x)+\varphi(y), x, y \in X$;

(iv) $\varphi(2 x)=2 \varphi(x), x \in X$,

and such that $d(x, y)=\varphi(x-y), x, y \in X$.

Conversely, if $\varphi: X \rightarrow \mathbb{R}$ satisfies (i)-(iv), then $d(x, y):=\varphi(x-y)$, $x, y \in X$, is a metric on $X$ and satisfies $(*)$.

Proof. Take $\varphi(x):=2 d((x / 2), 0), x \in X$. Then it follows from $(*)$ that $\varphi(x-y)=d(x, y)$. Conditions (i)-(iv) follow easily from (1.1) and (1.2). For the converse, it is easy to check that conditions (i), (ii) and (iii) imply that $d$ is a metric, and (*) follows from (iv).

It is well known that conditions (iii) and (iv) imply

$$
\varphi(n x)=n \varphi(x), \quad x \in X, n \in \mathbb{N} .
$$

Hence, $\varphi(p x)=p \varphi(x), x \in X, p \in \mathbb{Q}_{+}$and, from (ii), one gets

$\left(\right.$ iv $\left.^{\prime}\right) \varphi(p x)=|p| \varphi(x), x \in X, p \in \mathbb{Q}$.

Proposition 2.2. Let $X$ be a real vector space, and let a mapping $\varphi: X \rightarrow[0, \infty)$ satisfy conditions (i)-(iv). Then $\varphi$ is a norm on $X$ if and only if $\varphi$ additionally satisfies

(v) for all $x \in X$ there exists $\varepsilon>0$ and $M \geq 0$ for all $t \in[0, \varepsilon]$, $\varphi(t x) \leq M$.

Proof. Obviously, each norm satisfies (v). Conversely, assuming (v), we have to check that $\varphi(t x)=|t| \varphi(x)$ for all $x \in X$ and $t \in \mathbb{R}$. Let $x \in X$ and $t \in \mathbb{R}$. For $n \in \mathbb{N}$, choose $r_{n} \in \mathbb{Q}$ such that $t-\varepsilon / n \leq r_{n} \leq t$. Thus, $0 \leq n\left(t-r_{n}\right) \leq \varepsilon$, which implies $0 \leq \varphi\left(\left(t-r_{n}\right) x\right) \leq M / n$. By (iii) and $\left(\mathrm{iv}^{\prime}\right)$,

$$
\varphi(t x) \geq \varphi\left(r_{n} x\right)-\varphi\left(\left(r_{n}-t\right) x\right)=\left|r_{n}\right| \varphi(x)-\varphi\left(\left(r_{n}-t\right) x\right),
$$

whence, letting $n \rightarrow \infty, \varphi(t x) \geq|t| \varphi(x)$. Inserting $t x$ and $1 / t$ in place of $x$ and $t$, respectively, we get the reverse inequality.

Remark 2.3. Actually, the above elementary proof can be omitted in view of a more general result. It follows from (iii) and (iv) that, 
for any fixed $x \in X$, the mapping $\varphi_{x}: \mathbb{R} \ni t \mapsto \varphi(t x) \in[0, \infty)$ is Jensen-convex and, due to (v), bounded from above on some interval $[0, \varepsilon]$. Hence, it is continuous (Bernstein-Doetsch theorem, cf., [1] or [2, Theorem 6.4.2]) and homogeneity of $\varphi_{x}$, with respect to real scalars, follows. What is more, intervals $[0, \varepsilon]$ on which mappings $\varphi_{x}$ are bounded from the above, can be replaced by sets from a wider class $\mathfrak{A}$ of subsets possessing the property that any $J$-convex function bounded from above on such set must be continuous (cf., [2, Chapter 9, Theorem 9.3.3]). Furthermore, one could replace (v) by any other condition guaranteeing continuity of $J$-convex mappings $\varphi_{x}$.

Let $D$ be a subset of a real vector space $X$. An algebraic interior of $D$ is defined by

$$
\begin{gathered}
x_{0} \in \text { alg int } D \Longleftrightarrow \text { for all } y \in X \text { there exists } \varepsilon>0: \\
\qquad x_{0}+\lambda y \in D \text { for } \lambda \in[0, \varepsilon) .
\end{gathered}
$$

We say that $D$ is bounded on rays at a point $x_{0} \in D$ whenever, for any $y \in X$, the set

$$
D_{y}:=\left\{x_{0}+\lambda y: \lambda \geq 0\right\} \cap D
$$

is bounded (however, the bound may depend on $y$ ).

Finally, we arrive at our main result.

Theorem 2.4 (Main theorem). Let $d$ be a metric in a real vector space $X$ satisfying the mid-segment property $(*)$. Suppose that there exist a subset $D \subset X$ and a point $x_{0} \in \operatorname{alg}$ int $D$ such that $D$ is bounded on rays at $x_{0}$. Then $d$ is generated by a norm.

Proof. Due to Proposition 2.1, the mapping $\varphi(x):=2 d((x / 2), 0)$ satisfies (i)-(iv) and generates $d$. Assuming that $D$ is bounded on rays at $x_{0} \in \operatorname{alg} \operatorname{int} D$ and defining $D^{\prime}:=D-x_{0}$, one gets that $0 \in \operatorname{alg}$ int $D^{\prime}$ and $D^{\prime}$ is bounded on rays at 0 . Thus, $\varphi$ satisfies (v), and it follows from Proposition 2.2 that $\varphi$ is a norm.

In particular, the following holds true. 
Corollary 2.5. Let $d$ be a metric in $X$ satisfying $(*)$, and suppose that there exists a bounded subset $D \subset X$ with a nonempty algebraic interior. Then $d$ is generated by a norm.

\section{REFERENCES}

1. F. Bernstein and G. Doetsch, Zur Theorie der konvexen Funktionen, Math. Ann. 76 (1915), 514-526.

2. M. Kuczma, An introduction to the theory of functional equations and inequalities. Cauchy's equation and Jensen's inequality, 2nd edition, Attila Gilányi, ed., Birkhäuser, Basel 2009.

3. T. Oikhberg and H. Rosenthal, A metric characterization of normed linear spaces, Rocky Mountain J. Math. 37 (2007), 597-608.

4. P. Šemrl, A characterization of normed spaces, J. Math. Anal. Appl. 343 (2008), 1047-1051.

5. _ A characterization of normed spaces among metric spaces, Rocky Mountain J. Math. 41 (2011), 293-298.

Institute of Mathematics, Pedagogical University of Cracow, PodchorąŻYCH 2, 30-084 Kraków, Poland

Email address: jacek@up.krakow.pl 